\title{
Electroweak Higgs production in Standard Model effective field theory at next-to-leading order in QCD
}

\author{
Céline Degrande \\ CERN, Theory Division, Geneva 23 CH-1211, Switzerland \\ E-mail: celine.degrande@cern.ch \\ Benjamin Fuks \\ UPMC Univ. Paris 06, Sorbonne Universités, UMR 7589, LPTHE, 75005 Paris, France \\ E-mail: fuks@lpthe.jussieu.fr
}

\section{Kentarou Mawatari}

Laboratoire de Physique Subatomique et de Cosmologie, Université Grenoble-Alpes, CNRS/IN2P3, 53 Avenue des Martyrs, 38026 Grenoble, France

E-mail: kentarou.mawatarilipsc.in2p3.fr

\section{Ken Mimasu*}

Center for Cosmology, Particle Physics and Phenomenology - CP3, Université Catholique de

Louvain, Louvain-la-neuve, Belgium

E-mail: ken.mimasu@uclouvain.be

\section{Verónica Sanz}

Department of Physics and Astronomy, University of Sussex, Brighton BN1 9QH, UK

E-mail: v.sanzesussex.ac.uk

\begin{abstract}
We study the effect of dimension-six operators of the standard model effective field theory relevant for vector-boson fusion and associated Higgs boson production at the LHC. We present predictions at the next-to-leading order accuracy in QCD that include matching to parton showers and that rely on fully automated simulations. We show the importance of the subsequent reduction of the theoretical uncertainties in improving the possible discrimination between effective field theory and standard model results and investigate the impact of including the squared contributions of the new operators given current constraints from global fits to low energy and LHC data. The difference between including and not including these formally higher order contributions was found to be significant in the associated production case, suggesting that an effective field theory interpretation of current measurements lies on the edge of its validity range.
\end{abstract}

XXV International Workshop on Deep-Inelastic Scattering and Related Subjects

3-7 April 2017

University of Birmingham, $U K$

${ }^{*}$ Speaker. 


\section{Introduction}

The LHC Run I and early Run II data have not yet put forward any strong evidence of physics beyond the standard model (SM) and limits on new states have instead been pushed to higher and higher energies. As we approach the limits of the so-called 'energy frontier', searches for deviations from SM interactions via precise measurements are becoming increasingly relevant and, indeed, perhaps represent the most likely source of evidence for new physics at the LHC. The effective field theory (EFT) extension of the SM (SMEFT), built from the SM symmetries and degrees of freedom by adding higher-dimensional operators to the SM Lagrangian is a well-defined, modelindependent parametrisation of these low energy effects of heavy new physics. It also possesses the advantage of a concrete matching procedure to map constraints from the Wilson coefficients of the SMEFT to the underlying parameters of theories beyond the SM.

The formulation of the effective Lagrangian truncated at canonical dimension six relies on the definition of a complete and non-redundant operator basis [1-6] and should additionally include the translations among the possible choices [7]. Moreover, since it parametrises small deviations from the SM, precise theoretical predictions are required both in the SM and in the SMEFT frameworks. As the accumulated data at the LHC grows and searches become increasingly precise as well as systematics dominated, the inclusion of higher-order corrections and their implementation in Monte Carlo (MC) event generators becomes essential to gain accuracy and control said uncertainties.

These proceedings present NLO+PS (next-to-leading order plus parton-shower) accurate predictions for electroweak Higgs-boson production at the LHC i.e. Higgs boson production in association with a weak boson $(\mathrm{VH})$ and via vector-boson fusion (VBF), including effects from a number of relevant SMEFT operators. In this work, based on [8], we consider five operators affecting VH. The first goal is to provide an independent implementation of VH using the FEYNRULES [9], NLOCT [10] and MADGRAPH5_AMC@NLO (MG5_AMC) [11-14] programs. Second, the use of this framework allows us to also provide the first predictions for VBF in the SMEFT at NLO+PS accuracy, to which the same operators contribute, thanks to the flexibility of general purpose MC event generators such as MG5_AMC. Although we only present results for a couple of benchmark scenarios motivated by global fits the EFT parameters, predictions for any scenario can be obtained with our public Universal FEYNRULES Output (UFO) model [15] using MG5_AMC.

\section{Theoretical framework}

\subsection{Model description}

In the EFT framework, new physics is expected to appear at a scale, $\Lambda$, large enough so that the new degrees of freedom can be integrated out. The SM Lagrangian $\mathscr{L}_{\text {SM }}$ is supplemented by higher-dimensional operators, as an expansion in $\Lambda$. Truncating the series at dimension six, we focus on five specific, bosonic operators ${ }^{1}$, which are relevant to the VH and VBF processes, taken

\footnotetext{
${ }^{1}$ The relevant fermionic operators are also considered in, e.g., [16].
} 


\begin{tabular}{|c|l|c|l|}
\hline Eq. (2.2) & Our conventions & Eq. (2.2) & Our conventions \\
\hline$g_{h \gamma \gamma}$ & $a_{H}-\frac{e^{2} v}{\Lambda^{2}} \bar{c}_{B B}$ & $g_{h z \gamma}^{(2)}$ & $\frac{e^{2} v}{4 \hat{s}_{W} \hat{c}_{W} \Lambda^{2}}\left[\bar{c}_{H W}+\bar{c}_{W}-2\left(\bar{c}_{B B}+\bar{c}_{B}\right)\right]$ \\
$g_{h z z}^{(1)}$ & $\frac{e^{2} v}{2 s_{W}^{2} \hat{c}_{W}^{2} \Lambda^{2}}\left[\hat{c}_{W}^{2} \bar{c}_{H W}+2 \hat{s}_{W}^{2} \bar{c}_{H B}-2 \hat{s}_{W}^{4} \bar{c}_{B B}\right]$ & $g_{h w w}^{(1)}$ & $\frac{e^{2} v}{2 \hat{s}_{W}^{2} \Lambda^{2}} \bar{c}_{H W}$ \\
$g_{h z z}^{(2)}$ & $\frac{e^{2} v}{4 \hat{s}_{W}^{2} \hat{c}_{W}^{2} \Lambda^{2}}\left[\hat{c}_{W}^{2}\left(\bar{c}_{H W}+\bar{c}_{W}\right)+2 \hat{s}_{W}^{2}\left(\bar{c}_{H B}+\bar{c}_{B}\right)\right]$ & $g_{h w w}^{(2)}$ & $\frac{e^{2} v}{4 \hat{s}_{W}^{2} \Lambda^{2}}\left[\bar{c}_{H W}+\bar{c}_{W}\right]$ \\
$g_{h z z}^{(3)}$ & $\frac{g^{2} v}{2 \hat{c}_{W}^{2}}+\frac{e^{4} v^{3}}{8 \hat{s}_{\hat{S}}^{2} c_{W}^{4} \Lambda^{2}}\left[\hat{c}_{W}^{2} \bar{c}_{W}+2 \bar{c}_{B}\right]$ & $g_{h w w}^{(3)}$ & $\frac{g^{2} v}{2}$ \\
$g_{h z \gamma}^{(1)}$ & $a_{H}^{\prime}+\frac{e^{2} v}{4 \hat{s}_{W} \hat{c}_{W} \Lambda^{2}}\left[\bar{c}_{H W}-2 \bar{c}_{H B}+4 \hat{s}_{W}^{2} \bar{c}_{B B}\right]$ & & \\
\hline
\end{tabular}

Table 1: New physics effects in three-point interactions involving a Higgs boson and electroweak gauge bosons. The loop-induced SM contributions to the Higgs-boson couplings to two photons $a_{H}$ and to one $Z$-boson and one photon $a_{H}^{\prime}$ have been explicitly indicated.

from the strongly interacting light Higgs (SILH) basis $[5,17,18]^{2}$.

$$
\begin{aligned}
\mathscr{L}= & \mathscr{L}_{\mathrm{SM}}+\frac{g^{\prime 2}}{4 \Lambda^{2}} \bar{c}_{B B} \Phi^{\dagger} \Phi B_{\mu v} B^{\mu v}+\frac{i g}{2 \Lambda^{2}} \bar{c}_{W}\left[\Phi^{\dagger} T_{2 k} \overleftrightarrow{D}_{\mu} \Phi\right] D_{v} W^{k, \mu v}+\frac{i g^{\prime}}{2 \Lambda^{2}} \bar{c}_{B}\left[\Phi^{\dagger} \overleftrightarrow{D}_{\mu} \Phi\right] \partial_{v} B^{\mu v} \\
& +\frac{i g}{\Lambda^{2}} \bar{c}_{H W}\left[D_{\mu} \Phi^{\dagger} T_{2 k} D_{v} \Phi\right] W^{k, \mu v}+\frac{i g^{\prime}}{\Lambda^{2}} \bar{c}_{H B}\left[D_{\mu} \Phi^{\dagger} D_{v} \Phi\right] B^{\mu v}
\end{aligned}
$$

where $g$ and $g^{\prime}$ denote the coupling constants of $S U(2)_{L}$ and $U(1)_{Y}$ respectively. The Wilson coefficients $\bar{c}$ are free parameters, $T_{2 k}$ are the generators of $S U(2)$ in the fundamental representation and the Hermitian derivative operators and gauge field strength tensors are defined as in [8]. After electroweak symmetry breaking, the weak and hypercharge gauge eigenstates mix to the physical $W$-boson, $Z$-boson and the photon $A$. The neutral sector mixing defines the Weinberg angle, whose sine and cosine we denote as $\hat{s}_{W} \equiv \sin \hat{\theta}_{W}$ and $\hat{c}_{W} \equiv \cos \hat{\theta}_{W}$, respectively. The higher-dimensional operators of Eq. (2.1) induce a modification of the gauge boson kinetic terms, including a mixing between the neutral SU(2) and hypercharge gauge bosons that can be eliminated via field redefinitions and a corresponding shift in the two gauge couplings. The net effect is a modification of the relations between the EW input parameters and the remaining internal parameters.

In unitary gauge and rotating all field to the mass basis, the most general three-point interactions involving a single (physical) Higgs boson and a pair of electroweak gauge bosons at this order in canonical dimension are given by

$$
\begin{aligned}
\mathscr{L}_{\mathrm{hvv}}= & -\frac{1}{4} g_{h \gamma \gamma} A_{\mu v} A^{\mu v} h-\frac{1}{4} g_{h z z}^{(1)} Z_{\mu v} Z^{\mu v} h-g_{h z z}^{(2)} Z_{v} \partial_{\mu} Z^{\mu v} h+\frac{1}{2} g_{h z z}^{(3)} Z_{\mu} Z^{\mu} h-\frac{1}{2} g_{h z \gamma}^{(1)} Z_{\mu v} A^{\mu v} h \\
& -g_{h z \gamma}^{(2)} Z_{v} \partial_{\mu} A^{\mu v} h-\frac{1}{2} g_{h w w}^{(1)} W_{\mu v}^{+} W^{-\mu v} h-g_{h w w}^{(2)}\left[W_{v}^{+} \partial_{\mu} W^{-\mu v} h+\text { h.c. }\right]+g_{h w w}^{(3)} W_{\mu}^{+} W^{-\mu} h .
\end{aligned}
$$

Table 1 shows the relation between the couplings in Eq. (2.2) and the Wilson coefficients in Eq. (2.1).

\subsection{Benchmark selection}

Table 2 shows the marginalised limits on the Wilson coefficients of interest from a (LO) global fit to LEP and LHC Run I data performed in Ref. [19,20]. We use the limits to inform our two

\footnotetext{
${ }^{2}$ Although the $W$-boson mass $m_{W}$ and $v$ are usually used as expansion parameters in this basis, our model explicitly uses a cutoff scale $\Lambda$. For all our numerical results, we set $\Lambda=m_{W}$.
} 
benchmark scenarios in the Wilson coefficient space. Since electroweak precision observables strongly constrain a particular linear combination of the $\bar{c}_{W}$ and $\bar{c}_{B}$ Wilson coefficients beyond a precision than can be hoped for at the LHC, we impose the relation $\bar{c}_{B}=-\bar{c}_{W} / 2$, such that the EFT effects correspond to the region in parameter space for which the LHC is capable of providing complementary information.

\begin{tabular}{|cc|cccc|}
\hline Coefficients & Bounds & Benchmarks & $\bar{c}_{H W}$ & $\bar{c}_{W}$ & $\bar{c}_{B}$ \\
\hline$\frac{m_{W}^{2}}{\Lambda^{2}}\left(\frac{1}{2} \bar{c}_{W}-\bar{c}_{B}\right)$ & {$[-0.035,0.005]$} & $\mathrm{A}: g_{h v v}^{(1)}, g_{h v v}^{(2)} \neq 0$ & 0.03 & 0 & 0 \\
$\frac{m_{W}^{2}}{\Lambda^{2}}\left(\frac{1}{2} \bar{c}_{W}+\bar{c}_{B}\right)$ & {$[-0.0033,0.0018]$} & $\mathrm{B}: g_{h v v}^{(1)} \neq 0, g_{h v v}^{(2)}=0$ & 0.03 & -0.03 & 0.015 \\
$\frac{m_{W}^{2}}{\Lambda^{2}} \bar{c}_{H W}$ & {$[-0.07,0.03]$} & & & & \\
$\frac{m_{W}^{2}}{\Lambda^{2}} \bar{c}_{H B}$ & {$[-0.045,0.075]$} & & & & \\
\hline
\end{tabular}

Table 2: Current $95 \%$ confidence level constraints on the considered effective coefficients marginalised in a global fit to LEP and LHC Run I data [19] (left). EFT benchmark points used in this study, with $\Lambda=m_{W}$ (right). We additionally set $\bar{c}_{B B}=\bar{c}_{H B}=0$ for simplicity.

In benchmark $A$, we only switch on the $\mathscr{O}_{H W}$ operator, inducing new physics contributions through both the $g_{h v v}^{(1)}$ and $g_{h v v}^{(2)}$ structures (see Table 1). With benchmark $B$, we additionally fix $\bar{c}_{W}$ to an equal and opposite value which turns on solely the $g_{h v v}^{(1)}$ coupling.

\section{Results}

We generate events for $13 \mathrm{TeV}$ LHC collisions using the LO and NLO NNPDF2.3 set of parton densities [21] for LO and NLO simulations respectively. Events are then showered and hadronised with Pythia8 [22], which is also used to decay the Higgs-boson. The Higgs branching fractions are rescaled according to predictions from EHDECAY [23]. Event reconstruction and analysis are performed with MADANALYSIS5 [24], which uses jet algorithms implemented in FASTJET [25]. Jets are defined using the anti- $k_{T}$ algorithm [26] with a radius parameter of 0.4. A 9-point scale variation envelope is used to estimate theoretical uncertainties around the central scale choices of $\mu_{0}=H_{T} / 2$ and $m_{W}$ for the VH and VBF processes, respectively ${ }^{3}$.

For the VH process, we have the Higgs decay into $b \bar{b}$ and a leptonic $W^{+}$decay. We impose that both $b$-jets and leptons have a pseudorapidity, $\eta$, and a transverse momentum, $p_{T}$, satisfying $|\eta|<2.5$ and $p_{T}>25 \mathrm{GeV}$ respectively, while non- $b$-tagged jets are required within $|\eta|<4$, for the same $p_{T}$ requirement. We select events by demanding the presence of one lepton and two $b$-jets based on truth-level hadronic information. In the case of VBF, we require at least two jets with $|\eta|<4.5$ and $p_{T}>25 \mathrm{GeV}$, and additionally impose that the Higgs boson decays into a pair of photons with $|\eta|<2.5$ and $p_{T}>20 \mathrm{GeV}$. We moreover include a standard VBF selection on the invariant mass $M_{j j}>500 \mathrm{GeV}$ and pseudorapidity separation $\Delta \eta_{j j}>3$ of the pair of forward jets.

In Fig. 1, we present the transverse momentum and pseudorapidity distributions of the $b \bar{b}$ system and the transverse mass of the charged lepton and $b \bar{b}$ systems for the VH process. In the

\footnotetext{
${ }^{3} H_{T}$ is defined at the parton-level as the scalar sum of the transversemomentum of all visible final-state particles and the missing transverse energy.
} 
VBF case, the transverse momentum of the diphoton system and the pseudorapidity and azimuthal angular separation of the dijet system are shown. We plot in the first two subfigures the relative difference between the SM predictions both considered benchmark points $\mathrm{A}$ and $\mathrm{B}, \delta_{S M}^{i}=\frac{\sigma_{i}}{\sigma_{S M}}-1$. Both LO+PS and NLO+PS results are shown including scale uncertainties. In the last subfigure, we show differential $K$-factors defined as the NLO/LO ratio taking only the total NLO uncertainty into account.
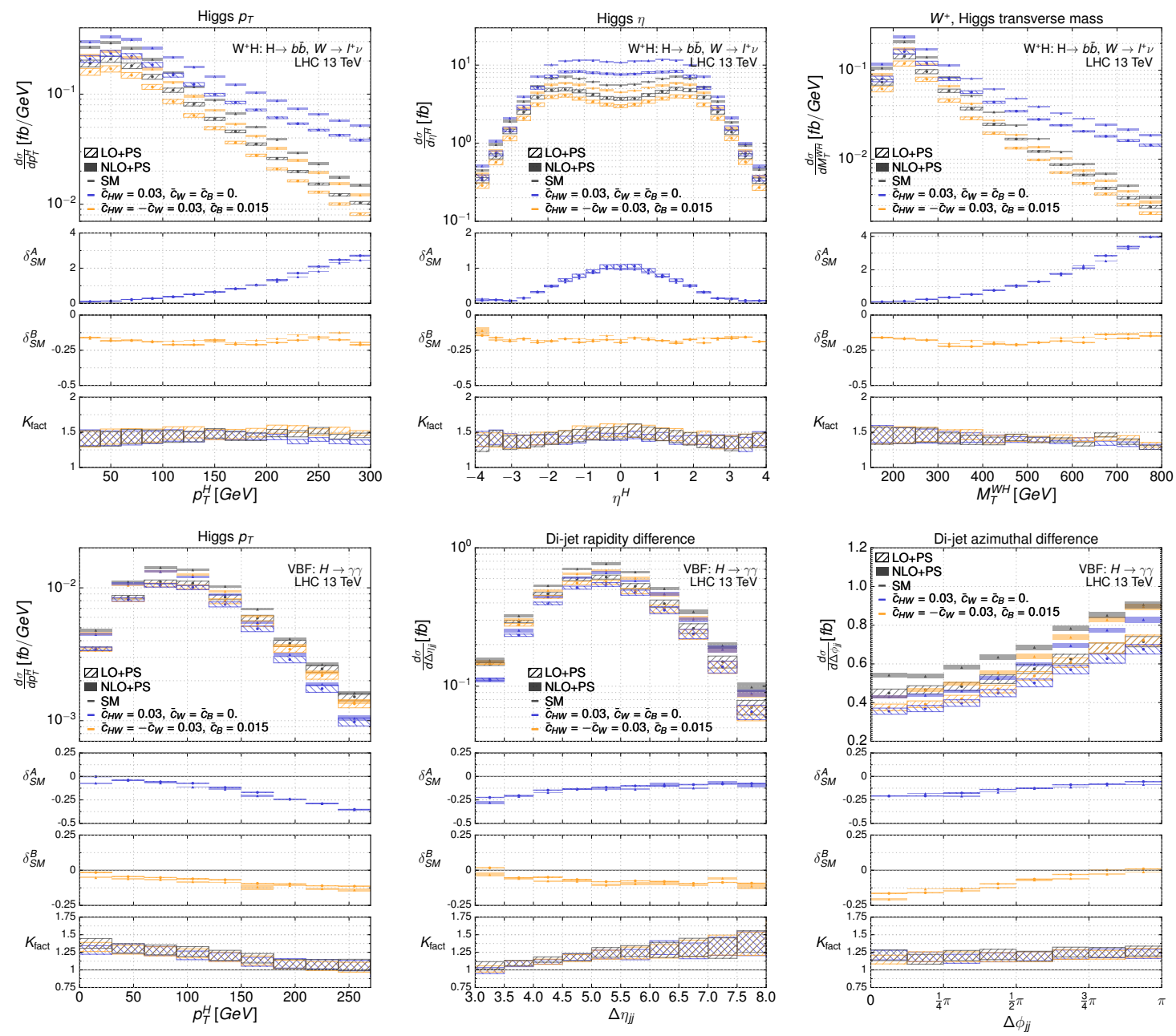

Figure 1: Differential distributions at the (N)LO+PS accuracy for the SM and the our benchmark scenarios. The upper and lower rows correspond to the $W H$ and VBF processes, respectively.

The $W H$ process is found to be stable under radiative corrections, as expected for any process with a Drell-Yan-like topology. $K$-factors are relatively flat and independent of the EFT parameters. Corrections are similar for the VBF process, with the exception of mildly kinematic-dependent $K$ factors for observables measuring the properties of hard jets. LO predictions do not overlap with the NLO results even after considering scale variation uncertainties. This behaviour is once again expected for processes that does not depend on $\alpha_{S}$ at Born-level. All distributions strongly depend on the value of the EFT Wilson coefficients. For the adopted scenario A, significant enhancements are observed in the kinematic tails. In contrast, event rates are only rescaled by about $15 \%-20 \%$ 
with respect to the SM for the scenario B. This originates from the presence of only the $g_{h v v}^{(1)}$ Lorentz structure, which is known to yield a smaller impact than that of $g_{h v v}^{(2)}[27,28]$. We recover a mild sensitivity to this scenario in VBF, suggesting that correlating the production modes should enable the two Lorentz structures to be disentangled.

While such large enhancements can be exploited to obtain powerful constraints on the SMEFT Wilson coefficients, they do raise the question of the validity of the EFT approach at large momentum transfer [20,29-31]. As a naive check, we made use of MG5_AMC's ability to select only interference contributions (at LO) to assess the impact of the (formally higher order) squared EFT terms given our benchmark choices. For $W H$, depending on the observable, these can range from $40 \%$ to $100 \%$ on the interference-only prediction for the benchmark scenario A, while they are much milder for the benchmark scenario B. This suggests that current sensitivities on this region of the Wilson coefficient parameter space may not yet lend themselves to an EFT interpretation within the validity of the framework. A reduction of the production rate from the SM value, as seen for benchmark scenario B, moreover indicates the dominance of the interference term between the SM and EFT contributions given that the squared terms are positive-definite. We observe such depletions across the board for VBF, with differences reaching at most $5 \%-12 \%$ for benchmark $A$ and remaining smaller for benchmark $B$

\section{Conclusions}

We have presented FEYNRULES and UFO implementations of dimension-six SMEFT operators affecting electroweak Higgs-boson production, which can be used for NLO(QCD)+PS accurate Monte Carlo event generation within the MG5_AMC framework. We have showcased the implementation by simulating both associated VH and VBF Higgs-boson production at the $13 \mathrm{TeV} \mathrm{LHC}$, selecting a pair of benchmark scenarios informed both by recent limits from global fits to the LEP and LHC Run I data and by theoretical motivations originating from integrating out certain popular ultraviolet realisations. We have found that EFT predictions and deviations from the SM are stable under higher-order corrections but still lie close to the validity limit of the EFT expansion. Overall, we have also observed a significant reduction of the theoretical errors, which would have an impact on the future measurements aiming to unravel dimension-six operator contributions. Our results exhibit an interesting complementarity of the two Higgs production channels since the interference pattern between the SM and the SMEFT contributions is quite different and benchmark-dependent.

\section{References}

[1] C. Burges and H. J. Schnitzer, Virtual Effects of Excited Quarks as Probes of a Possible New Hadronic Mass Scale, Nucl.Phys. B228 (1983) 464.

[2] C. N. Leung, S. Love, and S. Rao, Low-Energy Manifestations of a New Interaction Scale: Operator Analysis, Z.Phys. C31 (1986) 433.

[3] W. Buchmuller and D. Wyler, Effective Lagrangian Analysis of New Interactions and Flavor Conservation, Nucl.Phys. B268 (1986) 621.

[4] B. Grzadkowski, M. Iskrzynski, M. Misiak, and J. Rosiek, Dimension-Six Terms in the Standard Model Lagrangian, JHEP 1010 (2010) 085, [arXiv: 1008 . 4884]. 
[5] R. Contino, M. Ghezzi, C. Grojean, M. Muhlleitner, and M. Spira, Effective Lagrangian for a light Higgs-like scalar, JHEP 1307 (2013) 035, [arXiv:1303.3876].

[6] R. S. Gupta, A. Pomarol, and F. Riva, BSM Primary Effects, Phys. Rev. D91 (2015), no. 3 035001, [arXiv:1405.0181].

[7] A. Falkowski, B. Fuks, K. Mawatari, K. Mimasu, F. Riva, and V. Sanz, Rosetta: an operator basis translator for Standard Model effective field theory, Eur. Phys. J. C75 (2015), no. 12 583, [arXiv:1508.05895].

[8] C. Degrande, B. Fuks, K. Mawatari, K. Mimasu and V. Sanz, Electroweak Higgs boson production in the standard model effective field theory beyond leading order in QCD, Eur. Phys. J. C77 (2017), no. 4 262, [arXiv:1609.04833].

[9] A. Alloul, N. D. Christensen, C. Degrande, C. Duhr, and B. Fuks, FeynRules 2.0 - A complete toolbox for tree-level phenomenology, Comput. Phys. Commun. 185 (2014) 2250-2300, [arXiv:1310.1921].

[10] C. Degrande, Automatic evaluation of $U V$ and R2 terms for beyond the Standard Model Lagrangians: a proof-of-principle, Comput. Phys. Commun. 197 (2015) 239-262, [arXiv: 1406.3030 ].

[11] N. D. Christensen, P. de Aquino, C. Degrande, C. Duhr, B. Fuks, M. Herquet, F. Maltoni, and S. Schumann, A Comprehensive approach to new physics simulations, Eur. Phys. J. C71 (2011) 1541, [arXiv:0906.2474].

[12] C. Degrande, C. Duhr, B. Fuks, D. Grellscheid, O. Mattelaer, and T. Reiter, UFO - The Universal FeynRules Output, Comput. Phys. Commun. 183 (2012) 1201-1214, [arXiv:1108.2040].

[13] P. de Aquino, W. Link, F. Maltoni, O. Mattelaer, and T. Stelzer, ALOHA: Automatic Libraries Of Helicity Amplitudes for Feynman Diagram Computations, Comput. Phys. Commun. 183 (2012) 2254-2263, [arXiv:1108.2041].

[14] J. Alwall, R. Frederix, S. Frixione, V. Hirschi, F. Maltoni, O. Mattelaer, H. S. Shao, T. Stelzer, P. Torrielli, and M. Zaro, The automated computation of tree-level and next-to-leading order differential cross sections, and their matching to parton shower simulations, JHEP 07 (2014) 079, [arXiv:1405.0301].

[15] http://feynrules.irmp.ucl.ac.be/wiki/HELatNLO.

[16] S.-F. Ge, H.-J. He, and R.-Q. Xiao, Probing New Physics Scales from Higgs and Electroweak Observables at $e^{+} e^{-}$Higgs Factory, arXiv:1603.03385.

[17] G. Giudice, C. Grojean, A. Pomarol, and R. Rattazzi, The Strongly-Interacting Light Higgs, JHEP 0706 (2007) 045, [hep-ph/ 0703164$].$

[18] A. Alloul, B. Fuks, and V. Sanz, Phenomenology of the Higgs Effective Lagrangian via FEYNRULES, JHEP 1404 (2014) 110, [arXiv: 1310 .5150].

[19] J. Ellis, V. Sanz, and T. You, The Effective Standard Model after LHC Run I, JHEP 03 (2015) 157, [arXiv:1410.7703].

[20] J. Ellis, V. Sanz, and T. You, Complete Higgs Sector Constraints on Dimension-6 Operators, JHEP 07 (2014) 036, [arXiv:1404.3667].

[21] NNPDF Collaboration, R. D. Ball, V. Bertone, S. Carrazza, L. Del Debbio, S. Forte, A. Guffanti, N. P. Hartland, and J. Rojo, Parton distributions with QED corrections, Nucl. Phys. B877 (2013) 290-320, [arXiv:1308.0598]. 
[22] T. Sjostrand, S. Mrenna, and P. Z. Skands, A Brief Introduction to PYTHIA 8.1, Comput. Phys. Commun. 178 (2008) 852-867, [arXiv: 0710 . 3820].

[23] R. Contino, M. Ghezzi, C. Grojean, M. Mühlleitner, and M. Spira, eHDECAY: an Implementation of the Higgs Effective Lagrangian into HDECAY, Comput.Phys.Commun. 185 (2014) 3412-3423, [arXiv:1403.3381].

[24] E. Conte, B. Fuks, and G. Serret, MadAnalysis 5, A User-Friendly Framework for Collider Phenomenology, Comput.Phys.Commun. 184 (2013) 222-256, [arXiv:1206.1599].

[25] M. Cacciari, G. P. Salam, and G. Soyez, FastJet User Manual, Eur. Phys. J. C72 (2012) 1896, [arXiv:1111.6097].

[26] M. Cacciari, G. P. Salam, and G. Soyez, The Anti-k(t) jet clustering algorithm, JHEP 04 (2008) 063, [arXiv:0802.1189].

[27] F. Maltoni, K. Mawatari, and M. Zaro, Higgs characterisation via vector-boson fusion and associated production: NLO and parton-shower effects, Eur. Phys. J. C74 (2014), no. 1 2710, [arXiv:1311.1829].

[28] K. Mimasu, V. Sanz, and C. Williams, Higher Order QCD predictions for Associated Higgs production with anomalous couplings to gauge bosons, JHEP 08 (2016) 039, [arXiv:1512.02572].

[29] A. Biekötter, A. Knochel, M. Krämer, D. Liu, and F. Riva, Vices and virtues of Higgs effective field theories at large energy, Phys. Rev. D91 (2015) 055029, [arXiv:1406.7320].

[30] C. Englert and M. Spannowsky, Effective Theories and Measurements at Colliders, Phys. Lett. B740 (2015) 8-15, [arXiv:1408.5147].

[31] R. Contino, A. Falkowski, F. Goertz, C. Grojean, and F. Riva, On the Validity of the Effective Field Theory Approach to SM Precision Tests, JHEP 07 (2016) 144, [arXiv: 1604.0644 4].

[32] ATLAS Collaboration, T. A. collaboration, Search for the bb decay of the Standard Model Higgs boson in associated W/ZH production with the ATLAS detector, .

[33] ATLAS Collaboration Collaboration, Search for the Standard Model Higgs boson produced in association with a vector boson and decaying to a b $\bar{b}$ pair in pp collisions at $13 \mathrm{TeV}$ using the ATLAS detector, Tech. Rep. ATLAS-CONF-2016-091, CERN, Geneva, Aug, 2016.

[34] J. M. Campbell and R. K. Ellis, An Update on vector boson pair production at hadron colliders, Phys. Rev. D60 (1999) 113006, [hep-ph/ 9905386$].$

[35] J. M. Campbell, R. K. Ellis, and C. Williams, Vector boson pair production at the LHC, JHEP $\mathbf{0 7}$ (2011) 018, [arXiv:1105.0020].

[36] J. M. Campbell, R. K. Ellis, and W. T. Giele, A Multi-Threaded Version of MCFM, Eur. Phys. J. C75 (2015), no. 6 246, [arXiv:1503.06182].

[37] S. Alioli, P. Nason, C. Oleari, and E. Re, A general framework for implementing NLO calculations in shower Monte Carlo programs: the POWHEG BOX, JHEP 06 (2010) 043, [arXiv: 1002 .2581].

[38] S. Frixione, P. Nason, and C. Oleari, Matching NLO QCD computations with Parton Shower simulations: the POWHEG method, JHEP 11 (2007) 070, [arXiv: 0709.2092 ]. 